\title{
Acknowledgment to Reviewers of Humanities in 2021
}

\section{Humanities Editorial Office}

Citation: Humanities Editorial Office. 2022. Acknowledgment to

Reviewers of Humanities in 2021.

Humanities 11: 22. https://doi.org/

10.3390/h11010022

Published: 28 January 2022

Publisher's Note: MDPI stays neutral with regard to jurisdictional claims in published maps and institutional affiliations.

Copyright: $\odot 2022$ by the author. Licensee MDPI, Basel, Switzerland. This article is an open access article distributed under the terms and conditions of the Creative Commons Attribution (CC BY) license (https://creativecommons.org/licenses/by/4.0/).

MDPI AG, St. Alban-Anlage 66, 4052 Basel, Switzerland

Rigorous peer-reviews are the basis of high-quality academic publishing. Thanks to the great efforts of our reviewers, Humanities was able to maintain its standards for the high quality of its published papers. Thanks to the contribution of our reviewers, in 2021, the median time to first decision was 31.4 days and the median time to publication was 64.5 days. The editors would like to extend their gratitude and recognition to the following reviewers for their precious time and dedication, regardless of whether the papers they reviewed were finally published:

Afoumado, Diane

Alexandre, Sandy

Alford, C. Fred

Aliaga-Lavrijsen, Jessica

Allan, Arlene

Allwork, Larissa

Al-Saber, Samer

Ambrozy, Marián

Anita, Jarzyna

Aragon, Janni

Aughterson, Kate

Baker, Timothy C.

Barnes, Diana

Barrios-Rubio, Andrés

Bashkin, Orit

Bayer, Gerd

Bayley, Annouchka

Bignell, Jonathan

Birns, Nicholas

Blinder, Caroline

Boccia, Flavio

Borgella, Alex

Botham, Paola

Braden, Laura

Branach-Kallas, Anna

Breu, Christopher

Brilli, Stefano

Broglio, Ronald

Brouillette, Sarah

Bryson, Michael

Burke, Verity

Burns, William

Cameron, Ed

Carver, Evan
Castelvedere, Antonella

Cavanagh, Sheila

Chakraborty, Abhik

Cinquegrani, Maurizio

Clement, Jennifer

Click, Melissa

Clinton, Esther

Coyer, Megan

Craemer, Thomas

Crome, Andrew

Daniel, O'Quinn

Davis, Doug

De Biasio, Anna

Dennis, Yeo Kah Sin

Derbyshire, Harry

Donato, Clorinda

Dove, Danielle Mariann

Dubino, Jeanne

Eaglestone, Robert

Eguíbar-Holgado, Miasol

Elbousty, Jonas

Encarnación-Pinedo, Estíbaliz

English, Rebecca

Erceg, Aleksandar

Erlmann, Veit

Ferrere, Alexandre

Finch, Helen

Finlay, Jayne

Follini, Tamara

Franks, Hallie

Friedline, Mary Leah

Ganeva, Mila

Garibaldi, Korey

Garloff, Katia 
Gherardi, Silvia

Ghoneim, Hala Ibrahim

Goddard, Lynette

Goldberg, Katherine

Gombár, Zsófia

González Ferrin, Emilio

Goodchild, Philip

Guidi, Simone

Hall, Ann C.

Hamilton, Lindsay

Hammar, Isak

Han, Lisa

Hatley, James

Haynes, Sarah F.

Heschel, Susannah

Hicok, Bethany

Hiltunen, Kaisa

Hogue, Lawrence

Holm, Nicholas

Horlor, Samuel

Horne, Philip

Howell, Edward

Hudson, James

Ilott, Sarah

Izzo, Donatella

Jansson, Mats

Jaussen, Paul

Johnsson, Henrik

Johnston, Allan

Jones, David

Jorgensen, Jeana

Kaneti, Marina

Keen, Paul

Kennedy, Rebecca Futo

Kilbourn, Russell J. A.

King, Edward

Klein, Jennie

Konarska-Zimnicka, Sylwia

Krase, Jerome

Krouk, Dean

Lamm, Kimberly Kay

Landwehr, Margarete J.

Lanser, Susan

Lauro, Sarah Juliet

Lázaro-Reboll, Antonio

Leal, Joanne

Lee, Christina

Lee, Wai Sum

Lesman, Robert

Leuschner, Eric

Lewis, Diane Wei
Lodge, Kirsten

Lombardi, Giancarlo

López Rodríguez, Marta Sofía

Lopez-Fernandez, Laura

López-Martínez, Gabriel

Lorre-Johnston, Christine

Luckhurst, Roger

MacKenzie, Garry

Maksymiuk, Gabriela

Malkmus, Bernhard

Malmio, Kristina

Marcin, Wołk

Marostica, Matt

Marquez, Xavier

Maxwell, Kristi

McConnell, Anne

Mcgirr, Elaine $\mathrm{M}$

McHardy, Fiona

Michael, John

Mihailescu, Dana

Miller, Timothy S.

Mills, Sophie

Miralles, Francesc

Morin, Emeline

Morrison, Susan

Morton, Lindsay

Muccini, Francesca

Mugerauer, Robert

Newson-Horst, Adele

Nicol, Poppy

Nutters, Daniel Rosenberg

Oishi, Eve

Onega Jaén, Susana

Packham, Jimmy

Pankratz, Anette

Panteli, Georgia

Parrinder, Patrick

Peacock, James

Phillips, W. Dana

Pires, Maria José Pereira

Pischedda, Pier

Plourde, Bruce

Pöhlmann, Sascha

Poole, Adrian

Prádanos-Garcia, Luis (Iñaki)

Prager, Brad

Prieto, Sara

Ramírez, Jesse

Rankin, Elizabeth

Rastas, Anna

Rawle, Steven 
Reddy, Maureen

Reinhard, Kathryn L.

Reynolds, Kendra

Ricci, Carlo

Riecken, Nils

Ritson, Katie

Rivers, Joseph

Roach, Joseph

Robinson, Edward

Robson, Margaret

Saito, Kumiko

Salmose, Niklas

Salö, Linus

Sandy, Mark

Sawczuk, Tomasz

Schiermer Andersen, Bjørn

Seavey, Ormond

Seeger, Sean

Selby, Nick

Seruya, Teresa

Shay, Marnee

Shem-Tov, Naphtaly

Sherri, Brown

Shimon, Dar

Sidorova, Elena

Silber, Stefan

Silverstein, Ben
Smith, Tom

Sobol, Joseph

Stähler, Axel

Stevens, Wren

Stiles, Emily

Strout, Irina Igorevna

Tabusso Marcyan, Ilaria

Takács, Levente

Tanchio, P. A.

Thornton, Kendell C.

Thue, Fredrik

Tierney, Orchid

Tiffany, Grace C.

Uboldi, Sara

Van Der Tuin, Iris

Veggian, Henry

Verstraten, Peter

Villalobos, Cristóbal Macías

Weiscott, Eric

Weston, Nathaniel Parker

Whyte, Zachary

Wilson, Joseph

Wylot, David

Yampolskaya, Anna

Zigarovich, Jolene

Zirker, Angelika

Zisser, Eyal 